\title{
DETERMINATION AND CO-ESTIMATE OF THE CHLORMADINONE ACETATE AND 17 $\alpha$-ETHINYL ESTRADIOL IN PHARMACEUTICAL FORMULATION AND DRINKING WATER SAMPLES BY DIGITAL DERIVATIVE SPECTOPHOTOMETRY
}

\author{
CÉSAR SOTO* ${ }^{*}$, ROMINA OTIPKA ${ }^{2}$, M. INÉS TORAL ${ }^{2}$, DIEGO PINO², \\ DAVID CONTRERAS ${ }^{I}$, JORGE YÁNEEZI
}

\author{
'Department of Analytical and Inorganic Chemistry, Faculty of Chemical Sciences, University of Concepción, PO Box: 160-C, Concepción, Chile. \\ ${ }^{*}$ Address correspondence to this author at the Department of Inorganic and Analytical Chemistry, Faculty of Chemical Sciences, \\ University of Concepción, PO Box: 160-C, Concepción, Chile. \\ ${ }^{2}$ Department of Chemistry, Faculty of Sciences, University of Chile, PO Box: 653, Santiago, Chile.
}

\begin{abstract}
S
In this work are presented two methods for the simultaneous determination of $17 \alpha$-ethinylestradiol (EEL) and chlormadinone acetate (CMA) by second order derivative spectrophotometry.

The first analytical method proposed is based in the dissolution and extraction of both drugs in acetonitrile. The solution was directly evaluated by second order derivative spectrophotometry with a smoothing factor of 8,000 and a scale factor of 10,000. The determination of EEL and CMA was carried out to 296,6 and $291.8 \mathrm{~nm}$, respectively. The detection limits (3.3 $\sigma$ criterion) for EEL and CMA were $6.9 \times 10^{-7}$ and $9.8 \times 10^{-8} \mathrm{~mol} / \mathrm{L}$, respectively.

Furthermore, a study of the effect of the excipients containing in the pharmaceutical formulation was included. Polyvidone presents greater tendency to produce interference, but its spectral bands are located between 215 and $240 \mathrm{~nm}$, so these bands do not interferes spectrally in the simultaneous determination of EEL and CMA. Both drugs were extracted from the pharmaceutical formulation with acetonitrile, obtaining a good recovery relative to the nominal content.

The second method is a screening method and was applied in fortified drinking water. In this method, drugs were extracted in chloroform, which was then removed with $\mathrm{N}_{2}$ and the residue was redissolved in acetonitrile. Due to the difference in the procedure in relation to the pharmaceutical formulation a new analytical parameters were obtained. For EEL this parameters were very similar to those obtained directly in solution, however these were higher for CMA, which could be attributed to that under these conditions was found a higher standard deviation of the blank. Recoveries of fortified samples were $81.8 \pm 1.5 \%$ and 101.1 $\pm 0.73 \%$ for EEL and CMA, respectively.
\end{abstract}

Keywords: $17 \alpha$-Ethinyl Estradiol; Chlormadinone Acetate; Derivative Spectrophotometry; Screening Method; Pharmaceutical Formulations.

\section{INTRODUCTION}

Diseases caused by hormonal changes involved in the menstrual and menopausal period committed a large portion of the world population. These conditions correspond to the syndromes premenstrual (PMS) and menopausic. Studies show that these disorders directly affect: Emotional Stability ${ }^{1}$, relationships and job performance ${ }^{2}$. PMS was initially treated with serotonergic inhibitors. Oral contraceptives have an alternative use controlling hormonal imbalances. Current drug development produced new formulations for use in the symptomatic treatment of PMS. 17- $\alpha$-estrogen ethinyl estradiol (EEL, Figure 1) is used in mixture with chlormadinone acetate (CMA, Figure 1) and medroxyprogesterone for treating this syndrome. CMA has antiandrogenic properties, decreasing peripheral effects of some masculinizing hormones. Both hormones belong to the so-called "next-generation contraceptives", its power lies in increasing their effects when combined. Combination contraceptives act primarily in the hypothalamus and pituitary, suppressing the gonadotropin secretion. Estrogens inhibit the release of follicle stimulating hormone and thus suppress follicular growth. Gestagen suppresses luteinizing hormone secretion, thereby preventing ovulation. Developing new drugs and formulations, involves the qualitative and quantitative analysis of these drugs in pharmaceutical formulations.

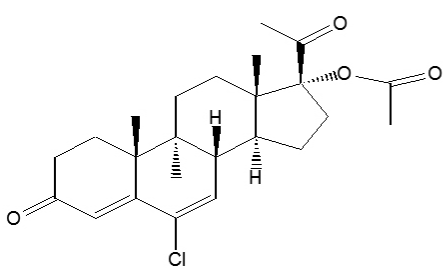

Chlormadinone Acetate (CMA)

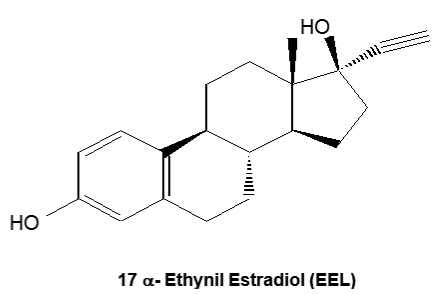

$17 \alpha$-Ethynil Estradiol (EEL)
Figure 1: Molecular Estructure for CMA y EEL.

The determinations of these drugs have been made with highcost techniques, requiring complicated previous steps. In this context, the simultaneous determination of EEL together with other drugs in pharmaceutical formulations has been reported by the following methods: Voltammetry, ${ }^{3,4}$ capillary electrophoresys ${ }^{5,6}$, HPLC-TMS $^{7}$, HPLC $^{8-10}$, derivative spectrophotometry ${ }^{11-16}$ and multivariate analysis based on the spectrophotometric data ${ }^{11,12}$. The simultaneous determinations of EEL and CMA in pharmaceutical formulations and water samples have not been reported. In this work, the derivative spectrophotometry technique was used, which in this moment is used when the spectral bands are overlapped. On the other hand, different rewiers, about methods that used this technique and its different modalities have been reported ${ }^{17-21}$.

On the other hands the past 20 years, various studies show that these drugs represent a new class of environmental contaminants. These drugs include antibiotics, hormones, analgesics, tranquilizers and chemotherapeutics. The pollution comes from deposition and urine, where a significant portion of the drug is eliminated from the body unmetabolized, furthermore of the improper disposal of waste of these products ${ }^{21}$. Several drugs have been found in surface water, groundwater and even drinking water ${ }^{22}$. The main routes of entry into the environment are those that involve human and veterinary medical treatments. The analytes excreted in urine and feces, may or may not metabolized (renal or liver) ${ }^{21}$. Then, these drugs enter to treatment plants wastewater through municipal sewer systems. Only has been reported the simultaneous determination of CMA and other drugs in environmental ${ }^{21}$ and biological ${ }^{22}$ samples. The first aim of this work was to develop, optimize and implement an analytical method for the simultaneous determination of EEL and CMA with UV-Visible derivative spectrophotometry, in pharmaceutical formulation. The second aim of this work was to develop, optimize and implement an screening method for the simultaneous determination of EEL and CMA with UV-visible spectrophotometry derivative, in drinking fortified water.

\section{EXPERIMENTAL}

\section{Instrumentation}

Shimadzu UV-1603 sprectrophotometer, software Version 3.7 (P/N 206-60570-04) and quartz cuvettes $(1 \mathrm{~cm})$ were used in absorption spectra measurements as function of 1 , using the solvent as blank. In order to obtain the second derivative in a range of 190-400 nm, $0.2 \mathrm{~nm}$ sampling interval and scanning rate of $480 \mathrm{~nm} / \mathrm{min}$, were used. All solid samples were weighed in a Sartorius R 200D balance with $0.01 \mathrm{mg}$ of uncertainty. 


\section{Reagents and Solutions}

EEL and CMA standards were purchased from Sigma-Aldrich ${ }^{\circledast}$. Stock solutions of concentration $2.0 \times 10^{-3} \mathrm{~mol} / \mathrm{L}$, were prepared by dissolving in $50 \mathrm{~mL}$ of acetonitrile, $40.5 \mathrm{mg}$ and $29.6 \mathrm{mg}$ of CMA and EEL, respectively. These solutions were diluted with the same solvent to obtain additional ranges of concentrations for the working solutions. The pharmaceutical product BELARA $^{\circledR}$, (Laboratorio Grunenthal, Stgo., Chile), containing both compounds was also dissolved using the same solvent. For, the study of solvent effects, CMA and EEL solutions were prepared by dissolving appropiate amounts of each drug in different solvents.

\section{Procedures}

\section{Photo-Stability Studies}

The photo-stability studies were carried out with individual $2.0 \times 10^{-5} \mathrm{~mol} / \mathrm{L}$ solutions of each analyte. These solutions were exposed to: dark, direct and indirect light. The zero order spectra of each drug were recorded every 30 minutes for 5 hours.

Calibration in solvent and simultaneous determination of EEL and CMA in synthetic samples and pharmaceutical formulation

Aliquots of stock solutions of EEL and CMA were diluted in acetonitrile to $10 \mathrm{~mL}$ in the range of $1.0 \times 10^{-5} \mathrm{~mol} / \mathrm{L}$ to $9.0 \times 10^{-5} \mathrm{~mol} / \mathrm{L}$. The calibration was carried out for each compound in the presence of $3.0 \times 10^{-5} \mathrm{~mol} / \mathrm{L}$ of CMA and EEL, respectively. The calibration were carried out with the above ranges of concentrations and the corresponding absolute values of DU, evaluated in second derivatives with smoothing factor value of 8,000 and amplification factor value of 10,000, at 291.8 and $296.6 \mathrm{~nm}$ for CMA and EEL, respectively.

For the simultaneous determination of EEL and CMA in synthetic samples the stock solutions of each drug were appropriately diluted to obtain solutions containing the following molar ratios: CMA : EEL of $1: 1$ to $5: 1$, corresponding to $8.0 \times 10^{-5} \mathrm{~mol} / \mathrm{L} / 8.0 \times 10^{-5} \mathrm{~mol} / \mathrm{L}$ and $4.0 \times 10^{-5} \mathrm{~mol} / \mathrm{L} / 8.0 \times 10^{-6}$ $\mathrm{mol} / \mathrm{L}$, respectively. For each solution, three replicates were performed. To then evaluate the second derivative spectra and calculate the concentrations of each compound, allowing the determination of the values for the \% recovery and relative standard deviation (RSD) for each drug.

For simultaneous determination of CMA and EEL in pharmaceutical formulation, ten tablets of "BELARA ${ }^{\mathbb{\Phi}}$ " (Lab. Grünenthal) were weighed and finely pulverized, then $91.5 \mathrm{mg}$ (average mass of the tablets) of the mixture was enriched with $19.7 \mathrm{mg}$ of EEL, and dissolved in $25 \mathrm{~mL}$ of acetonitrile and stirred for $15 \mathrm{~min}$ in an ultrasonic bath. The solution was centrifuged for 10 min at $5000 \mathrm{rpm}$, from the supernatant were extracted the followings aliquots $0.25,0.75,1.25,1.75$ and $2.25 \mathrm{~mL}$, which were dissolved to the mark of $5 \mathrm{~mL}$ with acetonitrile, obtaining the following ranges of concentrations: $1.0 \times 10^{-5}$ to $9.0 \times 10^{-5} \mathrm{~mol} / \mathrm{L}$ for CMA and $1.4 \times 10^{-5}$ to $1.2 \times 10^{-4} \mathrm{~mol} / \mathrm{L}$ for EEL.

On the other hand, the determination of the analytes in pharmaceutical formulations was carried out with the same procedure described above, for to obtain for mass CMA / EEL ratio of 10:1. In the average mass of the tablet was added $0.17 \pm 0.01 \mathrm{mg}$ of EEL, so as to obtain $9.0 \times 10^{-5} \mathrm{~mol} / \mathrm{L}$ for CMA and $1.2 \times 10^{-5} \mathrm{~mol} / \mathrm{L}$ for EEL.

\section{Calibration and determination of CMA and EEL in drinking water}

A solid mixture containing $4 \mathrm{mg}$ of each analyte and $800 \mathrm{mg}$ of $\mathrm{NaCl}$ in order to obtain lower concentrations were prepared. Different masses of the mixture were added to $50 \mathrm{~mL}$ water and stirred $(15 \mathrm{~min})$. Then were added $5 \mathrm{~mL}$ of chloroform to be separated in a separating funnel, the organic phase was extracted in glass beakers. This was evaporated with nitrogen, and the remaining solid was dissolved in $5 \mathrm{~mL}$ of acetonitrile, obtaining solutions between, $1.0 \times 10^{-5} \mathrm{~mol} / \mathrm{L}-9.0 \times 10^{-5} \mathrm{~mol} / \mathrm{L}$ for CMA and $1.4 \times 10^{-5}-1.2 \times 10^{-4} \mathrm{~mol} / \mathrm{L}$ for EEL.

In the determination of CMA and EEL in fortified drinking water samples with CMA and EEL, a sample drinking water was collected from the campus taps located in the Analytical Lab at Chemistry Dept. This sample was filtered first in $45 \mu \mathrm{m}$ (PVDF membrane) and kept at $4^{\circ} \mathrm{C}$ until analysis. Then were treated as described above in the respective subsection and second derivative spectra were evaluated and the concentrations of each compound were calculated, allowing the determination of the corresponding values of recovery and relative standard deviation (RSD).

\section{RESULTS AND DISCUSSION}

\section{Qualitative Studies of Solubility}

Qualitative evaluation of the dissolving power of the solvents were carried out with solutions $1 \times 10^{-3} \mathrm{~mol} / \mathrm{L}$ for both drugs using the solvents shown in Table 1. Based on these results, acetonitrile was selected.

Table 1: Qualitative studies of solubility for CMA and EEL

\begin{tabular}{|c|c|c|c|c|}
\hline Drugs & Diethylether & Ethanol & Water & Acetonitrile \\
\hline CMA & + & + & - & +++ \\
\hline EEL & + & + & - & +++ \\
\hline
\end{tabular}

- Insoluble, + Slightly soluble, ++ Soluble, +++ Completely soluble

\section{Spectral Behavior.}

Individual solutions of both analytes were evaluated against blank (acetonitrile). Spectra of both drugs show overlap between 190 and $320 \mathrm{~nm}$ (Figure 2). CMA has a bandwidth corresponding to an absorption maximum between 250 and $320 \mathrm{~nm}$. EEL presents two absorption bands; a centered between 250 and $300 \mathrm{~nm}$, and other between 190 and $250 \mathrm{~nm}$, the last band is the highest. In this context, derivative spectrophotometry technique was used, for the simultaneous determination of these drugs.

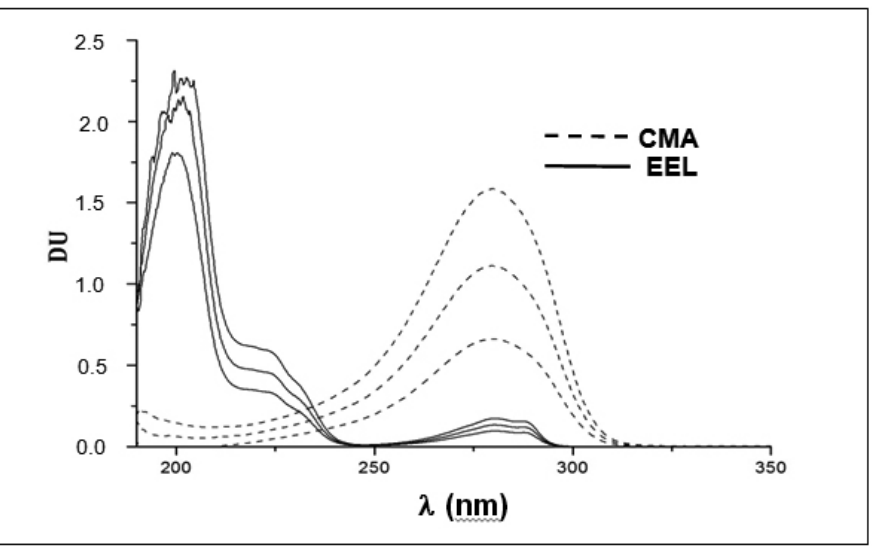

Figure 2: Zero order absorption spectra, of CMA (1.0x10-5, 3.0x10-5 and $5.0 \times 10-5 \mathrm{~mol} / \mathrm{L})$ and EEL $(1.4 \times 10-5,4.1 \times 10-5$ and $6.9 \times 10-5 \mathrm{~mol} / \mathrm{L})$, in acetonitrile for each drugs.

\section{Photostability studies}

To verify the stability of the analytes under study, solutions of $4.0 \times 10^{-5}$ $\mathrm{mol} / \mathrm{L}$ for both analytes were used separately. These solutions were exposed to: direct light (sunlight), indirect light (laboratory) and darkness and the spectrophotometric measurements were recorded every 30 minutes for $5 \mathrm{hrs}$. The spectral signals remain constant over the study period, indicating that none of the analytes undergoes chemical changes.

\section{Selection of spectral variables}

Derivative Order

This selection was carried out deriving the zero order spectra of each analyte, as according to Savitzky and Golay models ${ }^{23}$, using 8,000 and 10,000, as smoothing and amplification factors, respectively. In this type of derivation, when the order is increased, signals decrease and the noises is increased. The signals decrease in major proportion that the noise, for this reason the signal / noise ratio decreases, so that a good selection of the order of derivation, it is important to obtain accuracy and precision in the results. Third order has a zero-crossing at $291 \mathrm{~nm}$ for EEL and two for CMA at 295 and $288 \mathrm{~nm}$ (Figure 3 ), these wavelengths were discarded, because the noise in the CMA spectral signal at high concentrations decreases the accuracy of determination. In Fourth order, EEL has better spectral definition, that CMA. Moreover the derivatives of first and second order have adequate resolution for analytical objectives. At first derivative EEL can be determined by graphical method between 304 and $307 \mathrm{~nm}$, but no measurement areas for CMA are presented. In the secondorder derivatives are present two zero-crossing, one to CMA at $291.8 \mathrm{~nm}$ and another at $296.6 \mathrm{~nm}$ for EEL, allowing simultaneous quantitative analysis of these analytes. Based on this, the second derivative order with high definition and relationship between spectral signal, was selected. 


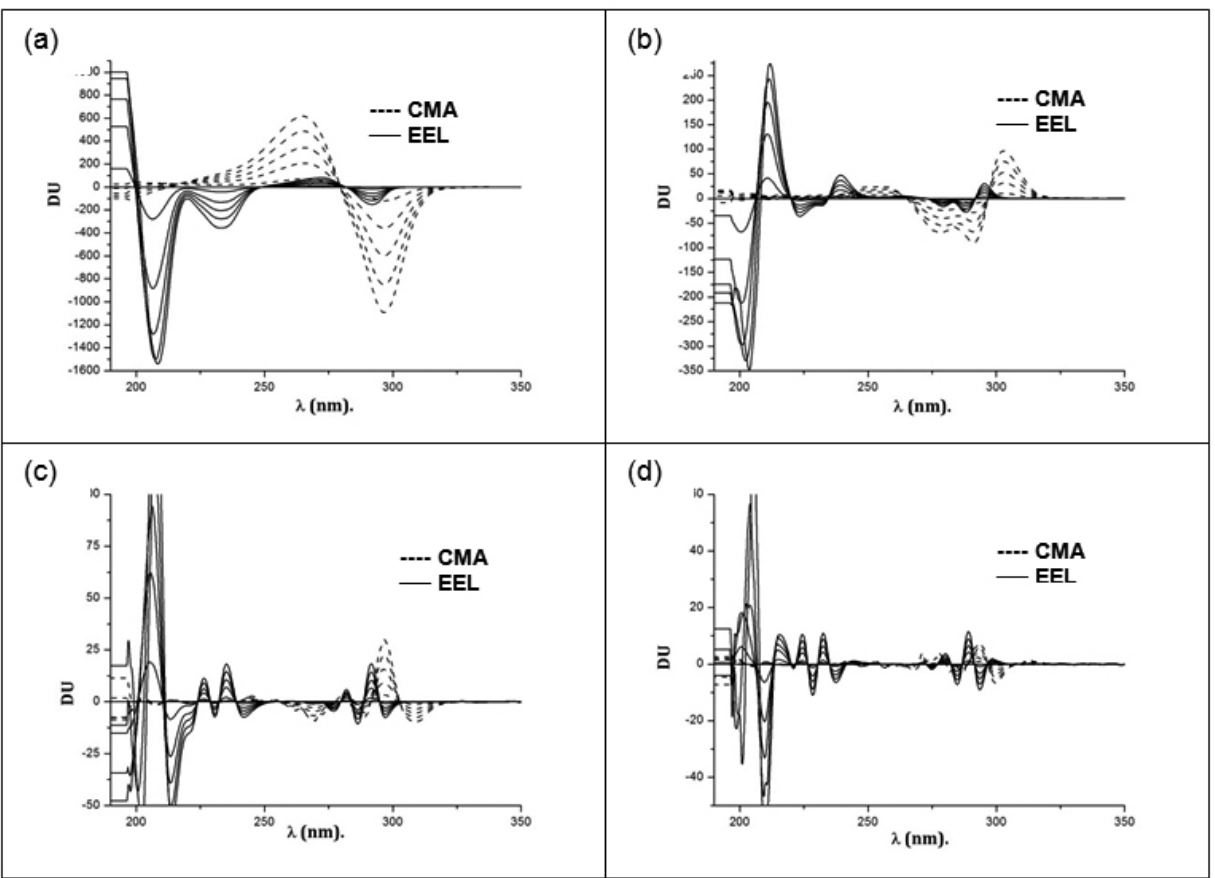

Figure 3: Derivative spectra of CMA and EEL (smoothing factor 8,000 and amplification factor 10,000). First order, (b) Second order, (c) Third order, (d) Fourth Order. Concentration range for both drugs: $1.0,3.0,5.0,7.0$ and $9.0 \times 10-{ }^{5} \mathrm{~mol} / \mathrm{L}$.

\section{Smoothing and Amplification Factors}

Using the second order derivation and 10,000 as the amplification factor, smoothing factor was optimized, this is related to the number of points used for the derivation of the zero order spectrum. The smoothing process and the intensity of the analytical signal are inversely related. For a $\Delta \lambda$ of $210 \mathrm{~nm}$, the smoothing factor presents the following values: $2,000,4,000,8,000$ and 16,000 . These values are given for default by the software, which depend on the $\Delta \lambda$ used. The value of the smoothing factor 8,000 , produced high signal/noise ratio, and for this reason was selected. The amplification factor only improves the lecture of the signal if distortion effect is not presented. Amplification factor 100 and 1,000 were discarded since they have lower intensity in the spectral signal, so that a 10,000 value was selected as optimal amplification factor.

\section{Analytical wavelength}

This selection was performed using, second-order derivation for both analytes individually, 8,000 and 10,000 as smoothing and amplification factors, respectively. At second-order, two zero-crossing are presented, one for CMA to $291.8 \mathrm{~nm}$ and the other for EEL at $296.6 \mathrm{~nm}$ (Figure 3B), the analytical signal is well defined (DU) and directly proportional to the concentration of the respective analyte. Based on these results the zero-crossings above mentioned, were selected for the simultaneous determination of CMA and EEL (Figure 4).

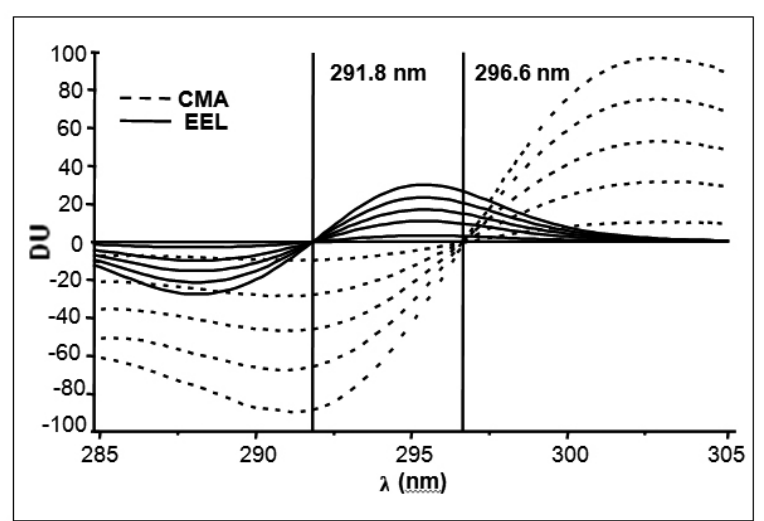

Figure 4: Second order derivative of CMA and EEL, separately, in acetonitrile. Concentration range for both drugs: 1.0, 3.0, 5.0, 7.0 and 9.0x10- ${ }^{5}$ $\mathrm{mol} / \mathrm{L}$.

\section{Analytical parameters for the calibration in solvent}

The calibration graphs binary mixtures of analytes, were performed using the optimal values of the spectral variables. A linear relationship between the concentrations of the analytes and their corresponding DU values, was observed. The analytical parameters were obtained by applying the Harmonized Tripartite Guideline ICH criteria ${ }^{24}$. DL and QL were obtained from: $3.3 \sigma / \mathrm{S}$ y $10 \sigma / \mathrm{S}$, respectively, with $\mathrm{S}$ as the slope of the calibration curve and $\sigma$ as the standard deviation, corresponding to the signal of 11 reagent blanks. Repeatability was expressed as relative standard deviation (RSD), and was obtained by measuring 9 standard samples containing the following mixture of EEL and CMA: $1.0 \times 10^{-5} \mathrm{~mol} / \mathrm{L} / 9.0 \times 10^{-5} \mathrm{~mol} / \mathrm{L}$. The application of this method allows obtaining, acceptable linearity, accurate and precise experimental results. These are shown in Table 2.

Table 2: Analytical parameters.

\begin{tabular}{|c|c|c|}
\hline $\begin{array}{c}\text { Analytical } \\
\text { parameters }\end{array}$ & CMA & EEL \\
\hline Linear Regression & $\mathrm{DU}=9.8 \times 10^{5} \mathrm{C}+1.43$ & $\mathrm{DU}=2.9 \times 10^{5} \mathrm{C}-0.37$ \\
\hline $\mathbf{D L}(\mathbf{m o l} / \mathbf{L})$ & $9.8 \times 10^{-8}$ & $6.9 \times 10^{-7}$ \\
\hline $\mathbf{Q L}(\mathbf{m o l} / \mathbf{L})$ & $2.9 \times 10^{-7}$ & $2.1 \times 10^{-6}$ \\
\hline $\mathbf{r}^{2}$ & 0.998 & 0.999 \\
\hline $\begin{array}{c}\text { Determination } \\
\text { Range (mol/L) }\end{array}$ & $2.9 \times 10^{-7}-9.0 \times 10^{-5}$ & $2.1 \times 10^{-6}-1.0 \times 10^{-3}$ \\
\hline $\begin{array}{c}\text { Analytical } \\
\text { wavelength (nm) }\end{array}$ & 291.8 & 296.6 \\
\hline RSD (\%) (11 blanks) & 2.9 & 0.7 \\
\hline
\end{tabular}

Recovery in Synthetic Samples

The recovery of both analytes in synthetic samples, allowed to evaluate the accuracy of the method. Between the relations $1: 1$ and 10:1, the values of recovery for both drugs are practically $100 \%$, this showing that not exist mutual interference between the analytes. Taking into account that the pharmaceutical formulation under study has a mass ratio for CMA / EEL of $66: 1$, equivalent to molar ratio of $50: 1$, the percentages of recovery were obtained in broad range, so that the feasibility of simultaneous determination directly in the pharmaceutical formulation samples. Nevertheless, recoveries in samples where the ratio of CMA :EEL is high, the percentages of recovery are greater than $100 \%$. In this context, it was decided to enrich the pharmaceutical 
formulation with a EEL standard solution, so that to obtain a molar ratio close to $1: 1$, considering that under these conditions the recoveries are favored for reaching practically $100 \%$.

The proportions for the accurate simultaneous determination of both analytes were established. The recoveries of both analytes are shown in Table 3.

Table 3. Recovery rates of CMA and EEL, in different proportions.

\begin{tabular}{|c|c|c|}
\hline CMA:EEL & $\begin{array}{c}\text { CMA } \\
\text { (\% recovery) }\end{array}$ & $\begin{array}{c}\text { EEL } \\
\text { (\% recovery) }\end{array}$ \\
\hline $1: 1$ & $100 \pm 2$ & $99 \pm 1$ \\
\hline $5: 1$ & $98 \pm 2$ & $97 \pm 2$ \\
\hline $8: 1$ & $102 \pm 2$ & $99 \pm 2$ \\
\hline $10: 1$ & $98 \pm 2$ & $97 \pm 2$ \\
\hline $14: 1$ & $106 \pm 2$ & $101 \pm 2$ \\
\hline $18: 1$ & $106 \pm 2$ & $100 \pm 2$ \\
\hline $20: 1$ & $112 \pm 3$ & $100 \pm 2$ \\
\hline $27: 1$ & $109 \pm 2$ & $101 \pm 2$ \\
\hline $40: 1$ & $110 \pm 4$ & $101 \pm 2$ \\
\hline $50: 1$ & $107 \pm 2$ & $98 \pm 2$ \\
\hline
\end{tabular}

\section{APPLICATION}

It was made using the optimal values for the spectral variables.

Extraction and Determination in Pharmaceutical Formulation

The excipients effect studies

The pharmaceutical formulation studied ("BELARA ${ }^{\circledR}$ ") containing nominally $0.3 \mathrm{mg}$ and $2.00 \mathrm{mg}$ of EEL and CMA, respectively (mass ratio, CMA:EEL=1:66.7), and mainly the following excipients: lactose monohydrate, corn starch, polyvidone $\mathrm{K} 30$, magnesium stearate, hypromellose, macrogol 6000 , propylene glycol, talc, titanium dioxide and red iron oxide. For these reason was necessary, study the spectral behavior of the excipients, evaluating possible interference. Polyvidone presents greater tendency to produce interference. Because of this, the polyvidone spectral behavior was studied. The classical spectral signal presents a broad band located between 215 and $240 \mathrm{~nm}$ (Figure 5a). Then this signal was processed with the optimal values of the spectral variables (Figure $5 \mathrm{~b}$ ). These signals do not interfere spectrally in the simultaneous determination of the analytes, because this was done at 291.8 and $296.6 \mathrm{~nm}$ for CMA and EEL, respectively.

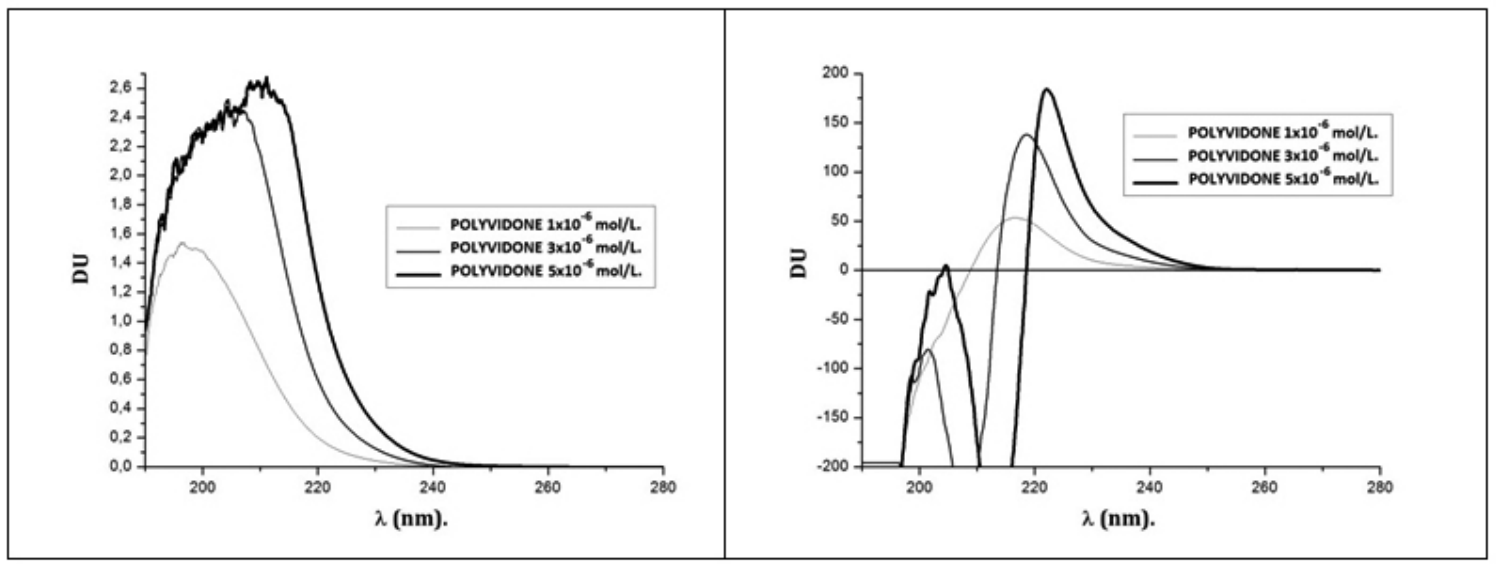

Figure 5: Polyvidone spectra: (a) Zero Order, (b) Second order derivative.

\section{Recovery in pharmaceutical formulation}

The respective process for the preparation of the samples was applied, taking into account the aforementioned mass ratio. The enrichment with EEL in the solid mixture, allowed to reduce the difference in mass between the two drugs, the new mass ratio was $1: 1$. Derivative spectra obtained show that it is possible the simultaneous determination of CMA and EEL in the selected analytical wavelengths. The results for the recoveries were, $1.90 \pm 0.08 \mathrm{mg}$ and $0.0310 \pm 0.0002 \mathrm{mg}$, for CMA and EEL, respectively. In both cases, no significant differences with the nominal content in the pharmaceutical formulation ("BELARA ${ }^{\circledR}$ ") were observed.

On the other hand, the determination of the analytes in pharmaceutical formulations was carried out with the same procedure described above, but using a mass CMA:EEL ratio of 10:1, the results for the recoveries were, $1.93 \pm$ $0.07 \mathrm{mg}$ and $0.0290 \pm 0.0002 \mathrm{mg}$, for CMA and EEL, respectively $1.96 \pm 0.08$ $\mathrm{mg}$ and $0.0310 \pm 0.0002 \mathrm{mg}$, for CMA and EEL Water

Analytical Parameters for the Calibration and Analysis of Drinking

For the determination of CMA and EEL in drinking water fortified, was necessary to extract the analytes with chloroform from this sample and then the extractant was removed with nitrogen, then the residue remaining containing the analytes was redissolved in acetonitrile, Owing to that process has several additional steps, in relation to the protocol used for the determination of both drugs in pharmaceutical formulations, using the procedure described in the respective section, new analytical parameters was obtained considering the RSD values of 11 blank. These parameters are shown in Table 4 . The analytical parameters in solvent and in drinking water for CMA are different, but for EEL are practically the same. The difference between the analytical parameters for CMA can be attributed mainly to the standard deviation of blanks, in the calibration in solvent are lower than in the proceedings in fortified drinking water.

The results obtained for the analysis in drinking water fortified samples, were added aliquots of stock solutions in order to obtain, $9.0 \times 10^{-5} \mathrm{~mol} / \mathrm{L}$ and $1.4 \times 10^{-5} \mathrm{~mol} / \mathrm{L}$ for CMA and EEL, respectively. The recoveries $(\mathrm{n}=5)$ obtained were: $81.8 \pm 1.5 \%$ and $101.1 \pm 0.73 \%$ for EEL and CMA, respectively.

Table 4: Analytical parameters in drinking water.

\begin{tabular}{|c|c|c|}
\hline $\begin{array}{c}\text { Analytical } \\
\text { parameters }\end{array}$ & CMA & EEL \\
\hline Linear Regression & $\mathrm{UD}=6.0 \times 10^{5} \mathrm{C}+1.5$ & $\mathrm{UD}=2.6 \times 10^{5} \mathrm{C}-2.1$ \\
\hline $\mathbf{D L}(\mathbf{m o l} / \mathbf{L})$ & $5.6 \times 10^{-7}$ & $7.3 \times 10^{-7}$ \\
\hline $\mathbf{Q L}(\mathbf{m o l} / \mathbf{L})$ & $1.7 \times 10^{-6}$ & $2.2 \times 10^{-6}$ \\
\hline $\begin{array}{c}\text { Determination } \\
\text { Range (mol/L) }\end{array}$ & $1.7 \times 10^{-7}-9.0 \times 10^{-5}$ & $2.2 \times 10^{-6}-1.0 \times 10^{-3}$ \\
\hline RSD (\%)(11 blanks) & 10.2 & 5.8 \\
\hline $\mathbf{r}^{2}$ & 0.998 & 0.996 \\
\hline
\end{tabular}

\section{CONCLUSIONS}

The spectral behavior of CMA and EEL is different and its bands are totally overlapped so that the derivative spectrophotometry is a good technique to develop a new method for determination of these drugs. The results 
obtained for the simultaneous determination of the binary mixture of CMA and EEL, by second derivative order were effective. The first method is an analytical method that can be a useful tool to the determination of EEL and CMA in the pharmaceutical formulation because the excipients do not affect in the simultaneous determination of both analytes. The simplicity, less timeconsuming and inexpensive of the proposed method allows rapid and precise quantification of EEL and CMA in pharmaceutical formulations. The second method, is one screening method, and was applied in fortified drinking water samples, with a recovery of $81.8 \pm 1.5 \%$ and $101.1 \pm 0.73 \%$ for EEL and CMA, respectively. After the extraction with chloroform, acetonitrile was selected as the dissolved solvent, obtained defined, reproducible and solved derived spectra and the simultaneous determination of both drugs.

\section{ACKNOWLEDGEMENTS}

The authors declare no conflict of interests and are grateful to the Dirección de Investigación de la Universidad de Concepción (DIUC) Project $\mathrm{N}^{\mathrm{o}}$ 210.021.029-10, and the Fondo Nacional de Investigación en Ciencia y Tecnología (FONDECYT), Project $\mathrm{N}^{\mathrm{o}} 1100103$, for the financial support.

\section{REFERENCES}

1. Easteal P.W., Aust. Inst. of Criminology, 31, 1, (1991).

2. D. Mishell, Am. J. Manag. Care, 11, 473, (2005).

3. E. Ghoneim, H. El-Desoky, M. Ghoneim, J. of Pharmaceutical and Biomedical Analysis 40, 255, (2006).

4. Chunya Li, Bioelectrochemistry, 70, 263, (2007).

5. V. Tripodi1, S. Flor, A. Carlucci and S. Lucangioli. Electrophoresis, 27, 4431, (2006).

6. Bin Du, Suyi Song, Xiufang Shi, and Zhenzhong Zhang. J. of Analytical Chemistry, 64, 59, (2009).
7. H. Licea-Perez, S. Wang, C. L. Bowen and E.Yang,. J. of Chromatography B, 852, 69, (2007).

8. A. Laban, S. Markovic, M. Stankov, P. Djurdjevic, Analytical letters, 37, 273, (2004).

9. A. Ahmed Ahn, S. Elgizawy, N. Omar, Analytical letters, 24, 2207, (1991).

10. M. Santoro, N. Kassab, M. Hasegawa, E. Kedor-Hackmann, Drug development and industrial pharmacy, 28, 741, (2002).

11. J. Berzas, J. Rodriguez, G. Castaneda, Analytica Chimica Acta, 340, 257, (1997).

12. J. Berzas, J. Rodriguez, G. Castaneda, Analytical Sciences, 13,1029, (1997).

13. E. Sour, H. Jalalizadeh, H. Farsam, Chemical \& pharmaceutical bulletin, 53, 949, (2005)

14. J. Nevado, J. Flores, G. Penalvo, F. Bernardo, Analytical letters, 30, 2221, (1997).

15. J. Berzas, J. Rodriguez, G. Castaneda, Analyst, 122, 41, (1997).

16. M. Toral, F. Nacaratte, F. Nova, R. Otipka, J.Chem Soc. Chil. 58, 1779, (2013).

17. J. Karpinska, Talanta 64, 801, (2004).

18. C. Bosch O., F. Sanchez R., Anal. Chim. Acta, 518, 1, (2004).

19. Y. Abdel-Aziz El-Sayed, N.A. El-Salem, Analytical Sciences, 21, 595, (2005).

20. F. Sánchez Rojas, C. Bosch Ojeda, Analytica Chimica Acta, 635, 22, (2009).

21. E.Malone, G.Dowling, Food additives and contaminants part A-chemistry analysis control exposure \& risk assessment, 26, 672, (2009).

22. L. Sun, W. Yong, X. Chu, J. Lin, J. of Chromatography A, 1216, 5416, (2009).

23. A.Savitzky and M. Golay. Anal. Chem. 36, 1627, (1964).

24. Validation of Analytical Procedures: Methodology (1997), International Conference on Harmonization of Technical Requirements for Registration of Pharmaceuticals for Human Use, Geneva, Switzerland, ICH Q2B. 